\title{
LIOUVILLE THEOREM FOR HEAT EQUATION ALONG ANCIENT SUPER RICCI FLOW VIA REDUCED GEOMETRY
}

\author{
KEITA KUNIKAWA AND YOHEI SAKURAI
}

\begin{abstract}
The aim of this article is to provide a Liouville theorem for heat equation along ancient super Ricci flow. We formulate such a Liouville theorem under a growth condition concerning Perelman's reduced distance.
\end{abstract}

\section{BACKGROUND}

In the present paper, we establish a Liouville theorem for heat equation along ancient super Ricci flow from Perelman's reduced geometric viewpoint (see Theorem 2.2 and Corollary 2.5 below). In this first section, we describe the background of our work.

1.1. Ancient super Ricci flow. We first recall the notion of ancient super Ricci flow. Let $(M, g(t))_{t \in I}$ be a smooth manifold equipped with a time-dependent Riemannian metric. Such a time-dependent Riemannian manifold is called Ricci flow if $g(t)$ evolves by

$$
\partial_{t} g=-2 \text { Ric . }
$$

The notion of Ricci flow has been established by Hamilton [17]. Perelman [32] has vastly developed Hamilton's Ricci flow theory, and solved the Poincaré conjecture. Since his celebrated work, the Ricci flow theory has been one of the central objects in geometric analysis.

A supersolution to the Ricci flow equation (1.1) is called super Ricci flow. More precisely, a time-dependent Riemannian manifold $(M, g(t))_{t \in I}$ is called super Ricci flow if

$$
\partial_{t} g \geq-2 \text { Ric . }
$$

Notice that static Riemannian manifold of non-negative Ricci curvature is a trivial example of super Ricci flow; in particular, super Ricci flow can be viewed as a time-dependent version of manifold of non-negative Ricci curvature. The notion of super Ricci flow has been introduced by McCann-Topping [30]. They have focused on the relation between Ricci flow theory and optimal transport theory, and characterized super Ricci flow in terms of the monotonicity of Wasserstein distance along heat flow. After that, several other characterizations have been investigated via Bochner inequality, Bakry-Émery gradient estimate, Poincaré inequality, logarithmic Sobolev inequality, and convexity of entropies (see e.g., [20], [28], [36]). Recently, based on such characterizations, the notion of super Ricci flow has been extended to timedependent (non-smooth) metric measure spaces by Sturm [36], and begun to be studied from metric measure geometric perspective (see e.g., [23], [24], [36]).

A Ricci flow $(M, g(t))_{t \in I}$ is said to be ancient when $I=(-\infty, 0]$. Ancient Ricci flow is one of the crucial objects in singular analysis of Ricci flow. Actually, it appears as a parabolic rescaling limit at singularity. In the present paper, we say that a super Ricci flow $(M, g(t))_{t \in I}$ is ancient when $I=(-\infty, 0]$.

Date: June 2, 2021.

2010 Mathematics Subject Classification. Primary 58J35; Secondly 53C44.

Key words and phrases. Ancient super Ricci flow; Heat equation; Liouville theorem; Space-only local gradient estimate. 
1.2. Liouville theorems for ancient solutions to heat equation. We next recall some previous works on Liouville theorems for ancient solutions to heat equation.

Liouville type properties for harmonic functions on Riemannian manifolds are some of the central topics in geometric analysis since the pioneering work by Yau [37] (cf. the recent survey [10] for its history). The classical Liouville theorem by Yau asserts that on a complete manifold of non-negative Ricci curvature, any positive harmonic functions must be constant.

One of the natural research directions is to extend such Liouville type properties to ancient solutions to heat equation. Here we recall that on a Riemannian manifold $(M, g)$, a solution $u: M \times I \rightarrow \mathbb{R}$ to the heat equation

$$
\partial_{t} u=\Delta u
$$

is called ancient when $I=(-\infty, 0]$. Souplet-Zhang [33] have established the following parabolic version of the Yau's Liouville theorem (see Theorem 1.2 in [33]):

Theorem 1.1 ([33]). Let $(M, g)$ be a complete Riemannian manifold of non-negative Ricci curvature. Then we have the following:

(1) Let $u: M \times(-\infty, 0] \rightarrow(0, \infty)$ be a positive ancient solution to the heat equation. If

$$
u(x, t)=\exp [o(d(x)+\sqrt{|t|})]
$$

near infinity, then $u$ must be constant. Here $d(x)$ denotes the Riemannian distance from a fixed point;

(2) let $u: M \times(-\infty, 0] \rightarrow \mathbb{R}$ be an ancient solution to the heat equation. If

$$
u(x, t)=o(d(x)+\sqrt{|t|})
$$

near infinity, then $u$ is constant.

The growth conditions (1.2) and (1.3) are sharp in the spatial direction (see [33]).

As stated in Subsection 1.1, super Ricci flow can be regarded as a time-dependent version of manifold of non-negative Ricci curvature. From this point of view, it seems to be natural to seek for Liouville theorems for heat equation along ancient super Ricci flow. More precisely, for an ancient super Ricci flow $(M, g(t))_{t \in(-\infty, 0]}$, the problem is to find suitable growth conditions for a solution $u: M \times(-\infty, 0] \rightarrow \mathbb{R}$ to heat equation

$$
\partial_{t} u=\Delta u
$$

such that $u$ must become constant.

In the study of ancient Ricci flow, we often work on the reverse time parameter

$$
\tau:=-t
$$

On this parameter, the above problem can be translated as follows: For a backward super Ricci flow $(M, g(\tau))_{\tau \in[0, \infty)}$, namely,

$$
\operatorname{Ric} \geq \frac{1}{2} \partial_{\tau} g
$$

the problem is to find suitable conditions for a solution $u: M \times[0, \infty) \rightarrow \mathbb{R}$ to backward heat equation

$$
\left(\Delta+\partial_{\tau}\right) u=0
$$

such that $u$ must become constant. Guo-Philipowski-Thalmaier [16] have approached this problem, and shown a Liouville theorem under a growth condition for entropy. Let us briefly 
recall their result. Let $(M, g(\tau))_{\tau \in[0, \infty)}$ be a complete backward super Ricci flow. For a fixed $x_{0} \in M$, let $k\left(x_{0}, x, \tau\right)$ stand for the heat kernel of the backward conjugate heat equation

$$
\left(\Delta-\partial_{\tau}-\frac{1}{2}\left(\operatorname{tr} \partial_{\tau} g\right)\right) v=0
$$

In [16], for a positive solution $u: M \times[0, \infty) \rightarrow(0, \infty)$ to backward heat equation, they have defined its entropy with respect to the heat kernel measure $k\left(x_{0}, x, \tau\right) m(x)$ by

$$
\mathcal{E}(\tau):=\int_{M}(u \log u)(x, \tau) k\left(x_{0}, x, \tau\right) d m(x) .
$$

They have shown the following (see Theorem 2 in [16]):

Theorem $1.2([16])$. Let $(M, g(\tau))_{\tau \in[0, \infty)}$ be a complete backward super Ricci flow. Let $u: M \times[0, \infty) \rightarrow(0, \infty)$ be a positive solution to backward heat equation. We assume

$$
\begin{aligned}
& \int_{M}\|\nabla(u \log u)\|^{2}(x, \tau) k\left(x_{0}, x, \tau\right) d m(x)<\infty, \\
& \int_{M}\left\|\nabla\left(\frac{\|\nabla u\|^{2}}{u}\right)\right\|^{2}(x, \tau) k\left(x_{0}, x, \tau\right) d m(x)<\infty
\end{aligned}
$$

for every $\tau>0$. If we have

$$
\lim _{\tau \rightarrow \infty} \frac{\mathcal{E}(\tau)}{\tau}=0
$$

then $u$ is constant.

The linear growth condition (1.6) is also sharp (see Example 1 in [16]).

Remark 1.3. In [16], the roles of $t$ and $\tau$ are reversed (cf. Remark 2 in [16]).

\section{Main Results}

In this section, we present our main result, and the key ingredient of its proof.

2.1. Liouville theorems via reduced geometry. We now state our Liouville theorem.

In [32, Perelman has discovered two kinds of important monotone quantities along Ricci flow. The one is entropy functionals called $\mathcal{F}$-functional and $\mathcal{W}$-functional, and the other is an integral quantity called reduced volume. Guo-Philipowski-Thalmaier [16] have introduced the growth condition (1.6) in Theorem 1.2 inspired by Perelman's entropy functionals (see Remark 1 in [16]). We aim to produce a reduced geometric counterpart of Theorem 1.2, We will prove a Liouville theorem under a growth condition for Perelman's reduced distance.

To state our main result, we recall some notions on a complete, time-dependent Riemannian manifold $(M, g(\tau))_{\tau \in[0, \infty)}$, which is not necessarily backward super Ricci flow. We define

$$
h:=\frac{1}{2} \partial_{\tau} g, \quad H:=\operatorname{tr} h .
$$

First, we briefly recall the notion of reduced distance (we explain contents of this paragraph in more detail in Subsection 3.1). For $(x, \tau) \in M \times(0, \infty)$, let $L(x, \tau)$ stand for the L-distance from a space-time base point $\left(x_{0}, 0\right)$, which is defined as the infimum of the so-called $\mathcal{L}$-length over all curves $\gamma:[0, \tau] \rightarrow M$ with $\gamma(0)=x_{0}$ and $\gamma(\tau)=x$. Then the reduced distance $\ell(x, \tau)$ is defined by

$$
\ell(x, \tau):=\frac{1}{2 \sqrt{\tau}} L(x, \tau) .
$$


In the static case of $g(\tau) \equiv g$, it holds that

$$
\ell(x, \tau)=\frac{d(x)^{2}}{4 \tau}
$$

for the Riemannian distance $d(x)$ from $x_{0}$ induced from $g$. We say that $(M, g(\tau))_{\tau \in[0, \infty)}$ is admissible if for every $\tau>0$ there is $c_{\tau} \geq 0$ depending only on $\tau$ such that $h \geq-c_{\tau} g$ on $[0, \tau]$. The admissibility ensures that the $L$-distance can be achieved by a minimal $\mathcal{L}$-geodesic.

Next, for a (time-dependent) vector field $V$, we recall the following Müller quantity $\mathcal{D}(V)$ (see Definition 1.3 in [31]), and trace Harnack quantity $\mathcal{H}(V)$ (see [18], Definition 1.5 in [31]):

$$
\begin{aligned}
& \mathcal{D}(V):=-\partial_{\tau} H-\Delta H-2\|h\|^{2}+4 \operatorname{div} h(V)-2 g(\nabla H, V)+2 \operatorname{Ric}(V, V)-2 h(V, V), \\
& \mathcal{H}(V):=-\partial_{\tau} H-\frac{H}{\tau}-2 g(\nabla H, V)+2 h(V, V) .
\end{aligned}
$$

Remark 2.1. Along general geometric flow, several geometric and analytic properties are known to hold under the non-negativity of Müller quantity. For instance, we possess the monotonicity of $\mathcal{W}$-functional (see Theorem 3.1 in [21] and Theorem 5.2 in [15]), and that of reduced volume (see Theorem 1.4 in [31] and Theorem 3.4 in [21]). We also have a differential Harnack inequality of Perelman type (see Corollary 9.3 in [32] and Theorem 1.2 in [5]), that of Cao type (see Theorem 1.3 in [4], Theorem 2.2 in [14] and Theorem 1.4 in [13]), and that of Cao-Hamilton type (see Theorem 1.1 in [6], Theorem 1.4 in [12] and Theorem 3.2 in [14]).

Now, let us consider a backward super Ricci flow $(M, g(\tau))_{\tau \in[0, \infty)}$. The backward super Ricci flow inequality (1.4) can be written as

$$
\text { Ric } \geq h .
$$

The static case of $h=0$ corresponds to the case where $(M, g(\tau))_{\tau \in[0, \infty)}$ is a complete manifold of non-negative Ricci curvature, and then $H=0$. The equality case of $h=$ Ric does the case where it is a complete backward Ricci flow, and then $H$ is equal to the scalar curvature. Our main result is the following:

Theorem 2.2. Let $(M, g(\tau))_{\tau \in[0, \infty)}$ be an admissible, complete backward super Ricci flow. We assume

$$
\mathcal{D}(V) \geq 0, \quad \mathcal{H}(V) \geq-\frac{H}{\tau}, \quad H \geq 0
$$

for all vector fields $V$. Then we have the following:

(1) Let $u: M \times[0, \infty) \rightarrow(0, \infty)$ be a positive solution to backward heat equation. If

$$
u(x, \tau)=\exp [o(\mathfrak{d}(x, \tau)+\sqrt{\tau})]
$$

near infinity, then $u$ is constant. Here $\mathfrak{d}(x, \tau)$ is defined by

$$
\mathfrak{d}(x, \tau):=\sqrt{4 \tau \ell(x, \tau)}
$$

(2) let $u: M \times[0, \infty) \rightarrow \mathbb{R}$ be a solution to backward heat equation. If

$$
u(x, \tau)=o(\mathfrak{d}(x, \tau)+\sqrt{\tau})
$$

near infinity, then $u$ is constant.

Regarding the assumption for $\mathcal{H}(V)$ in (2.3), the right hand side and the second term in the definition of $\mathcal{H}(V)$ cancel out each other. Also, the non-negativity of $H$ guarantees that of $\ell(x, \tau)$; in particular, $\mathfrak{d}(x, \tau)$ is well-defined.

Remark 2.3. In the static case of $h=0$, Theorem 2.2 is reduced to Theorem 1.1. Indeed, we have $H=0, \mathcal{D}(V)=\operatorname{Ric}(V, V), \mathcal{H}(V)=0$, and hence the admissibility and the assumption (2.3) automatically hold. Furthermore, the equality (2.1) yields $\mathfrak{d}(x, \tau)=d(x)$. 
Remark 2.4. For later convenience, we divide $\mathcal{D}(V)$ into two parts. We set

$$
\begin{aligned}
\mathcal{D}_{0}(V) & :=-\partial_{\tau} H-\Delta H-2\|h\|^{2}+4 \operatorname{div} h(V)-2 g(\nabla H, V), \\
\mathcal{R}(V) & :=\operatorname{Ric}(V, V)-h(V, V)
\end{aligned}
$$

such that $\mathcal{D}(V)=\mathcal{D}_{0}(V)+2 \mathcal{R}(V)$ (cf. Definition 1 in [22]). The backward super Ricci flow inequality (2.2) implies $\mathcal{R}(V) \geq 0$. In particular, if we have $\mathcal{D}_{0}(V) \geq 0$, then the assumption $\mathcal{D}(V) \geq 0$ in $(2.3)$ is satisfied.

We now observe the equality case of $h=$ Ric. Let $(M, g(\tau))_{\tau \in[0, \infty)}$ be a complete backward Ricci flow. Then we see $\mathcal{D}(V)=0$ since the evolution formula of scalar curvature along Ricci flow tells us that the sum of the first three terms of $\mathcal{D}_{0}(V)$ vanishes (see e.g., Corollary 4.20 in [1]), the contracted second Bianchi identity says that the remaining part of $\mathcal{D}_{0}(V)$ also does (see e.g., (2.16) in [1]), and the equality condition $h=$ Ric leads to $\mathcal{R}(V)=0$. Additionally, if we suppose the non-negativity of curvature operator, then the admissibility and $H \geq 0$ hold. Moreover, if we assume its boundedness, then the Hamilton's trace Harnack inequality for ancient Ricci flow yields the assumption for $\mathcal{H}(V)$ in (2.3) (see Corollary 1.2 in [18], and also Theorem 5.2 and Corollary 5.3 below). Thus, from Theorem 2.2 we conclude:

Corollary 2.5. Let $(M, g(\tau))_{\tau \in[0, \infty)}$ be a complete backward Ricci flow with bounded, nonnegative curvature operator. Then we have:

(1) Let $u: M \times[0, \infty) \rightarrow(0, \infty)$ be a positive solution to backward heat equation. If

$$
u(x, \tau)=\exp [o(\mathfrak{d}(x, \tau)+\sqrt{\tau})]
$$

near infinity, then $u$ is constant;

(2) let $u: M \times[0, \infty) \rightarrow \mathbb{R}$ be a solution to backward heat equation. If

$$
u(x, \tau)=o(\mathfrak{d}(x, \tau)+\sqrt{\tau})
$$

near infinity, then $u$ is constant.

Remark 2.6. From the viewpoint of the works by Bailesteanu-Cao-Pulemotov [3], Zhang [41, and Ecker-Knopf-Ni-Topping [11], it seems to be reasonable to consider growth conditions not for Riemannian distance $d(x, \tau)$ induced from $g(\tau)$ but for $\mathfrak{d}(x, \tau)$ in formulating a Liouville theorem of Souplet-Zhang type for solutions to backward heat equation along backward Ricci flow. We discuss this topic in Subsection 6.5.

2.2. Gradient estimates. We here present the key ingredient of the proof of Theorem 2.2.

For that purpose, we now recall the notion of $K$-super Ricci flow, which is a time-dependent version of Riemannian manifold whose Ricci curvature is bounded from below by $K$. For $K \in \mathbb{R}$, a time-dependent Riemannian manifold $(M, g(t))_{t \in I}$ is called $K$-super Ricci flow if

$$
\frac{1}{2} \partial_{t} g+\text { Ric } \geq K g \text {. }
$$

Here 0-super Ricci flow is nothing but super Ricci flow. When the equality in (2.8) holds, $(M, g(t))_{t \in I}$ is called $K$-Ricci flow.

Remark 2.7. In the context of metric measure geometry, the following general notion called $(K, N)$-super Ricci flow has been investigated: For $K \in \mathbb{R}$ and $N \in[n, \infty]$, a time-dependent weighted Riemannian manifold $(M, g(t), \phi(t))_{t \in I}$ is called $(K, N)$-super Ricci flow if

$$
\frac{1}{2} \partial_{t} g+\operatorname{Ric}_{\phi}^{N} \geq K g
$$

where $n$ is the dimension of $M$, and $\operatorname{Ric}_{\phi}^{N}$ denotes the so-called $N$-Bakry-Émery Ricci curvature associated with the time-dependent density function $\phi(t)$. We notice that $(K, N)$-super 
Ricci flow is a time-dependent version of weighted Riemannian manifold satisfying the condition that $N$-Bakry-Émery Ricci curvature is bounded from below by $K$, which is equivalent to the so-called curvature-dimension condition $\mathrm{CD}(K, N)$ in the sense of Sturm [34], 35] and Lott-Villani [29]. It seems that the notion of $(K, N)$-super Ricci flow firstly appeared in the work of Arnaudon-Coulibaly-Thalmaier [2] (see Theorem 4.1 (b) in [2]), where they only consider the case of $N=\infty$. After their work, it has been studied by Sturm [36], Kopfer-Sturm [24], Kopfer [23], or in a series of works done by Li-Li [25], 26], 27], [28].

We now consider a backward $K$-super Ricci flow $(M, g(\tau))_{\tau \in[0, \infty)}$, namely,

$$
\operatorname{Ric} \geq h+K g .
$$

The backward $K$-super Ricci flow inequality (2.9) leads to $\mathcal{R}(V) \geq K\|V\|^{2}$. Theorem 2.2 is a direct consequence of the following space-only local gradient estimate:

Theorem 2.8. For $K \geq 0$, let $(M, g(\tau))_{\tau \in[0, \infty)}$ be an $n$-dimensional, admissible, complete backward $(-K)$-super Ricci flow. We assume

$$
\mathcal{D}(V) \geq-2 K\left(H+\|V\|^{2}\right), \quad \mathcal{H}(V) \geq-\frac{H}{\tau}, \quad H \geq 0
$$

for all vector fields $V$. Let $u: M \times[0, \infty) \rightarrow(0, \infty)$ stand for a positive solution to backward heat equation. For $R, T>0$ and $A>0$, we suppose $u \leq A$ on

$$
Q_{R, T}:=\{(x, \tau) \in M \times(0, T] \mid \mathfrak{d}(x, \tau) \leq R\} .
$$

Then there exists a positive constant $C_{n}>0$ depending only on $n$ such that on $Q_{R / 2, T / 4}$,

$$
\frac{\|\nabla u\|}{u} \leq C_{n}\left(\frac{1}{R}+\frac{1}{\sqrt{T}}+\sqrt{K}\right)\left(1+\log \frac{A}{u}\right) .
$$

In the static case of $h=0$, Theorem 2.8 has been established by Souplet-Zhang [33] (see Theorem 1.1 in [33], and cf. Remark 2.3). We here recall that the gradient estimate in [33] is a parabolic version of the local gradient estimate of Cheng-Yau [7] for harmonic functions, and also inspired by the space-only global gradient estimate by Hamilton [19].

Remark 2.9. By the backward (-K)-super Ricci flow inequality (2.9), if we have $\mathcal{D}_{0}(V) \geq$ $-2 K H$, then the assumption for $\mathcal{D}(V)$ in (2.10) holds (cf. Remark 2.4).

In Section 5, we examine the trace Harnack inequality for $K$-Ricci flow, and derive the following result from Theorem 2.8 in the same manner as Corollary 2.5.

Corollary 2.10. For $K \geq 0$, let $(M, g(\tau))_{\tau \in[0, \infty)}$ be an n-dimensional, complete backward $(-K)$-Ricci flow with bounded, non-negative curvature operator. Let $u: M \times[0, \infty) \rightarrow(0, \infty)$ be a positive solution to backward heat equation. For $R, T>0$ and $A>0$, we suppose $u \leq A$ on $Q_{R, T}$. Then there is a positive constant $C_{n}>0$ depending only on $n$ such that on $Q_{R / 2, T / 4}$,

$$
\frac{\|\nabla u\|}{u} \leq C_{n}\left(\frac{1}{R}+\frac{1}{\sqrt{T}}+\sqrt{K}\right)\left(1+\log \frac{A}{u}\right) .
$$

In Section 6, we compare our gradient estimate with other space-only local gradient estimates obtained by Bailesteanu-Cao-Pulemotov [3], Zhang [41], Ecker-Knopf-Ni-Topping [11], and discuss the advantage of our work.

\section{REDUCED GEOMETRY}

To capture the behavior of Ricci flow, Perelman [32] has introduced the reduced geometry, which is the comparison geometry on his reduced distance. This section is devoted to review and study on reduced geometry. Throughout this subsection, let $(M, g(\tau))_{\tau \in[0, \infty)}$ stand for an $n$-dimensional, complete time-dependent Riemannian manifold. 
3.1. Basics of reduced geometry. We first review basics of reduced geometry. The references are [8], [31], [32], [38], [39], [40]. We mainly refer to the works of Yokota [39], [40], which is compatible with our setting.

We start with giving the precise definition of reduced distance. For a curve $\gamma:\left[\tau_{1}, \tau_{2}\right] \rightarrow M$, its $\mathcal{L}$-length is defined as

$$
\mathcal{L}(\gamma):=\int_{\tau_{1}}^{\tau_{2}} \sqrt{\tau}\left(H+\left\|\frac{d \gamma}{d \tau}\right\|^{2}\right) d \tau
$$

Notice that its critical point over all curves with fixed endpoints is known to be characterized by the following $\mathcal{L}$-geodesic equation:

$$
X:=\frac{d \gamma}{d \tau}, \quad \nabla_{X} X-\frac{1}{2} \nabla H+\frac{1}{2 \tau} X+2 h(X)=0 .
$$

For $(x, \tau) \in M \times(0, \infty)$, the $L$-distance $L(x, \tau)$ and reduced distance $\ell(x, \tau)$ from a space-time base point $\left(x_{0}, 0\right)$ are defined by

$$
L(x, \tau):=\inf _{\gamma} \mathcal{L}(\gamma), \quad \ell(x, \tau):=\frac{1}{2 \sqrt{\tau}} L(x, \tau),
$$

where the infimum is taken over all curves $\gamma:[0, \tau] \rightarrow M$ with $\gamma(0)=x_{0}$ and $\gamma(\tau)=x$. A curve is called minimal $\mathcal{L}$-geodesic from $\left(x_{0}, 0\right)$ to $(x, \tau)$ if it attains the infimum of (3.1).

We next discuss the admissibility introduced in Subsection 2.1. Recall that $(M, g(\tau))_{\tau \in[0, \infty)}$ is called admissible if for each $\tau>0$ there is $c_{\tau} \geq 0$ depending only on $\tau$ such that $h \geq-c_{\tau} g$ on $[0, \tau]$. Note that $h=$ Ric if it is backward Ricci flow. Perelman [32] has developed reduced geometry under uniform boundedness of sectional curvature. Ye [38] has shown that many fundamental parts of Perelman's reduced geometry, including $\mathcal{L}$-geodesic theory, still hold under a lower Ricci curvature bound. Furthermore, Yokota [39], [40] has pointed out that for general geometric flow that is not necessarily (backward) Ricci flow, the admissibility ensures such fundamental properties with the same proof as in 38] (cf. Section 2 in [39]).

We assume that $(M, g(\tau))_{\tau \in[0, \infty)}$ is admissible. Then for every $(x, \tau) \in M \times(0, \infty)$, there is at least one minimal $\mathcal{L}$-geodesic (see Proposition 2.8 in [38]). Also, the functions $L(\cdot, \tau)$ and $L(x, \cdot)$ are locally Lipschitz in $(M, g(\tau))$ and $(0, \infty)$, respectively (see Propositions 2.12 , 2.13, and also 2.14 in [38]); in particular, they are differentiable almost everywhere.

Let us state derivative formulas for reduced distance. In the rest of this section, we assume that $\ell$ is smooth at $(\bar{x}, \bar{\tau}) \in M \times(0, \infty)$. For such a point, the minimal $\mathcal{L}$-geodesic is uniquely determined, and its tangent vector $\bar{X}$ coincides with the gradient of $\ell$ (see Lemma 2.11 in [38]). We possess the following (see Subsection 2.3 in [39]):

Proposition 3.1. At $(\bar{x}, \bar{\tau})$ we have

$$
\begin{aligned}
\partial_{\tau} \ell & =H-\frac{\ell}{\bar{\tau}}+\frac{1}{2 \bar{\tau}^{3 / 2}} \mathcal{K}_{\mathcal{H}}, \\
\|\nabla \ell\|^{2} & =-H+\frac{\ell}{\bar{\tau}}-\frac{1}{\bar{\tau}^{3 / 2}} \mathcal{K}_{\mathcal{H}}, \\
\Delta \ell & \leq-H+\frac{n}{2 \bar{\tau}}-\frac{1}{2 \bar{\tau}^{3 / 2}} \mathcal{K}_{\mathcal{H}}-\frac{1}{2 \bar{\tau}^{3 / 2}} \mathcal{K}_{\mathcal{D}},
\end{aligned}
$$

where

$$
\mathcal{K}_{\mathcal{H}}:=\int_{0}^{\bar{\tau}} \tau^{3 / 2} \mathcal{H}(\bar{X}) d \tau, \quad \mathcal{K}_{\mathcal{D}}:=\int_{0}^{\bar{\tau}} \tau^{3 / 2} \mathcal{D}(\bar{X}) d \tau .
$$

Remark 3.2. The estimates (3.2), (3.3) have been stated in [39]. We can check them by the same calculations as that in the proof of $(7,88),(7.89)$ in [8] for backward Ricci flow. Also, a similar Laplacian comparison to (3.4) has been done in [39]. But in [39], its last term did 
not appear since he assumed the non-negativity of $\mathcal{D}_{0}(V)$ and $\mathcal{R}(V)$ defined as (2.6) and (2.7), which implies that of $\mathcal{D}(V)$, at first (see Assumption 2.1 in [39]). One can verify (3.4) by carefully following the calculations in the proof of (7.90) in [8].

Remark 3.3. For general geometric flow, Müller [31] has obtained similar inequalities to (3.2), (3.3), (3.4) under compactness of $M$ (see (5.18) in [31]). Here he has studied both forward and backward reduced distances in a unified way, and the backward one corresponds to our reduced distance. Moreover, he has shown that even if $\ell$ is not smooth at $(\bar{x}, \bar{\tau})$, they hold in the barrier sense (see Lemma 5.3 in [31]). By using the same barrier function constructed in the proof of Lemma 5.3 in [31], we can also conclude that even if $\ell$ is not smooth at $(\bar{x}, \bar{\tau})$, the inequalities (3.2), (3.3), (3.4) hold in the barrier sense. More precisely, there exist a sufficiently small $\delta>0$, an open neighborhood $U$ of $\bar{x}$ in $M$, and a smooth upper barrier function $\hat{\ell}$ at $(\bar{x}, \bar{\tau})$ on $U \times(\bar{\tau}-\delta, \bar{\tau}+\delta)$ (i.e., $\widehat{\ell} \geq \ell$, and the equality holds at $(\bar{x}, \bar{\tau}))$ such that $\widehat{\ell}$ satisfies (3.2), (3.3), (3.4) at $(\bar{x}, \bar{\tau})$.

For $(x, \tau) \in M \times(0, \infty)$ we define

$$
\bar{L}(x, \tau):=4 \tau \ell(x, \tau)=\mathfrak{d}(x, \tau)^{2} .
$$

Summing up (3.2) and (3.4) yields the following (see Section 2 in [40]):

Lemma 3.4. At $(\bar{x}, \bar{\tau})$ we have

$$
\left(\Delta+\partial_{\tau}\right) \bar{L} \leq 2 n-\frac{2}{\sqrt{\bar{\tau}}} \mathcal{K}_{\mathcal{D}}
$$

3.2. Key estimates. In this subsection, we will collect two key estimates for the proof of our main results. Let $(M, g(\tau))_{\tau \in[0, \infty)}$ stand for an $n$-dimensional, admissible, complete timedependent Riemannian manifold. Continuing from the above subsection, we also assume that the reduced distance is smooth at $(\bar{x}, \bar{\tau}) \in M \times(0, \infty)$. We first note the following:

Lemma 3.5. Let $K \geq 0$. We assume

$$
\mathcal{D}(V) \geq-2 K\left(H+\|V\|^{2}\right), \quad H \geq 0
$$

for all vector fields $V$. Then at $(\bar{x}, \bar{\tau})$ we have

$$
\left(\Delta+\partial_{\tau}\right) \bar{L} \leq 2 n+2 K \bar{L} .
$$

Proof. Lemma 3.4 together with the assumption and $\bar{L}=2 \sqrt{\bar{\tau}} L$ tells us that

$$
\begin{aligned}
\left(\Delta+\partial_{\tau}\right) \bar{L} & \leq 2 n-\frac{2}{\sqrt{\bar{\tau}}} \mathcal{K}_{\mathcal{D}} \leq 2 n+\frac{4 K}{\sqrt{\bar{\tau}}} \int_{0}^{\bar{\tau}} \tau^{3 / 2}\left(H+\|\bar{X}\|^{2}\right) d \tau \\
& \leq 2 n+4 K \sqrt{\bar{\tau}} \int_{0}^{\bar{\tau}} \tau^{1 / 2}\left(H+\|\bar{X}\|^{2}\right) d \tau=2 n+4 K \sqrt{\bar{\tau}} L=2 n+2 K \bar{L} .
\end{aligned}
$$

This proves the lemma.

We also prove that under the trace Harnack inequality and the non-negativity of $H$, the norm of the gradient of $\mathfrak{d}$ is bounded by a universal constant (cf. (2.54) in [38]).

Lemma 3.6. We assume

$$
\mathcal{H}(V) \geq-\frac{H}{\tau}, \quad H \geq 0
$$

for all vector fields $V$. Then at $(\bar{x}, \bar{\tau})$ we have

$$
\|\nabla \mathfrak{d}\|^{2} \leq 3
$$


Proof. From the trace Harnack inequality in (3.5) it follows that

$$
\mathcal{K}_{\mathcal{H}} \geq-\int_{0}^{\bar{\tau}} \sqrt{\tau} H d \tau \geq-L=-2 \sqrt{\bar{\tau}} \ell
$$

By the non-negativity of $H$, and by applying (3.6) to (3.3), we obtain

$$
\|\nabla \ell\|^{2} \leq\|\nabla \ell\|^{2}+H=\frac{\ell}{\bar{\tau}}-\frac{1}{\bar{\tau}^{3 / 2}} \mathcal{K}_{\mathcal{H}} \leq \frac{3 \ell}{\bar{\tau}} .
$$

This is equivalent to the desired one.

Remark 3.7. This estimate seems to be natural since in the static case of $h=0$, the function $\mathfrak{d}$ coincides with the Riemannian distance function from the fixed point, whose norm of the gradient is equal to a universal constant 1 (see Remark 2.3).

\section{Proof of main Results}

We now prove Theorem 2.8, and conclude Theorem 2.2. We give a proof of Theorem 2.8 by using the key estimates obtained in Subsection 3.2 along the line of the proof of SoupletZhang gradient estimate in [33] (for comparison between our method of the proof and that in [33], see Subsection 6.1 below). Throughout this section, let $(M, g(\tau))_{\tau \in[0, \infty)}$ stand for an $n$-dimensional, admissible, complete time-dependent Riemannian manifold.

4.1. Backward heat equations. In the next two subsections, we will make preparations towards the proof of Theorem 2.8. Here, we examine properties of a positive solution $u$ : $M \times[0, \infty) \rightarrow(0, \infty)$ to backward heat equation. We begin with the following:

Lemma 4.1. We set

$$
f:=\log u \text {. }
$$

Then we have

$$
\begin{aligned}
\left(\Delta+\partial_{\tau}\right) f & =-\|\nabla f\|^{2}, \\
\left(\Delta+\partial_{\tau}\right)\|\nabla f\|^{2} & =2\left\|\nabla^{2} f\right\|^{2}-2 g\left(\nabla\|\nabla f\|^{2}, \nabla f\right)+2 \mathcal{R}(\nabla f),
\end{aligned}
$$

where $\mathcal{R}(\nabla f)$ is defined as (2.7).

Proof. From direct calculations and backward heat equation (1.5), we deduce

$$
\Delta f=-\frac{\|\nabla u\|^{2}}{u^{2}}+\frac{\Delta u}{u}=-\frac{\|\nabla u\|^{2}}{u^{2}}-\frac{\partial_{\tau} u}{u}=-\|\nabla f\|^{2}-\partial_{\tau} f
$$

and hence (4.2). The second one (4.3) follows from

$$
\begin{aligned}
\partial_{\tau}\|\nabla f\|^{2} & =-\left(\partial_{\tau} g\right)(\nabla f, \nabla f)+2 g\left(\nabla \partial_{\tau} f, \nabla f\right) \\
& =-2 \operatorname{Ric}(\nabla f, \nabla f)-2 g(\nabla \Delta f, \nabla f)-2 g\left(\nabla\|\nabla f\|^{2}, \nabla f\right)+2 \mathcal{R}(\nabla f) \\
& =-\Delta\|\nabla f\|^{2}+2\left\|\nabla^{2} f\right\|^{2}-2 g\left(\nabla\|\nabla f\|^{2}, \nabla f\right)+2 \mathcal{R}(\nabla f) .
\end{aligned}
$$

Here we used a fundamental formula for time-dependent metrics (see e.g., Lemma 4.5 in [1]), the equation (4.2), and Bochner formula. This completes the proof.

We next show the following:

Lemma 4.2. Let $f$ be defined as (4.1). We assume $\sup f<1$, and set

$$
w:=\frac{\|\nabla f\|^{2}}{(1-f)^{2}}
$$

Then we have

$$
\left(\Delta+\partial_{\tau}\right) w-\frac{2 f g(\nabla w, \nabla f)}{1-f} \geq 2(1-f) w^{2}+\frac{2 \mathcal{R}(\nabla f)}{(1-f)^{2}}
$$


Proof. By straightforward computations,

$$
\nabla w=\frac{\nabla\|\nabla f\|^{2}}{(1-f)^{2}}+\frac{2\|\nabla f\|^{2} \nabla f}{(1-f)^{3}}
$$

and hence

$$
g(\nabla w, \nabla f)=\frac{g\left(\nabla\|\nabla f\|^{2}, \nabla f\right)}{(1-f)^{2}}+\frac{2\|\nabla f\|^{4}}{(1-f)^{3}}=\frac{g\left(\nabla\|\nabla f\|^{2}, \nabla f\right)}{(1-f)^{2}}+2(1-f) w^{2} .
$$

Furthermore, (4.5) yields

$$
\begin{aligned}
\Delta w & =\frac{\Delta\|\nabla f\|^{2}}{(1-f)^{2}}+\frac{4 g\left(\nabla\|\nabla f\|^{2}, \nabla f\right)}{(1-f)^{3}}+\frac{6\|\nabla f\|^{4}}{(1-f)^{4}}+\frac{2\|\nabla f\|^{2} \Delta f}{(1-f)^{3}} \\
& =\frac{2 g(\nabla w, \nabla f)}{1-f}+\frac{2\|\nabla f\|^{4}}{(1-f)^{4}}+\frac{2 g\left(\nabla\|\nabla f\|^{2}, \nabla f\right)}{(1-f)^{3}}+\frac{\Delta\|\nabla f\|^{2}}{(1-f)^{2}}+\frac{2\|\nabla f\|^{2} \Delta f}{(1-f)^{3}} .
\end{aligned}
$$

Likewise, Lemma 4.1 and (4.5) imply

$$
\begin{aligned}
\partial_{\tau} w & =\frac{\partial_{\tau}\|\nabla f\|^{2}}{(1-f)^{2}}+\frac{2\|\nabla f\|^{2} \partial_{\tau} f}{(1-f)^{3}} \\
& =-\frac{2\|\nabla f\|^{4}}{(1-f)^{3}}-\frac{2 g\left(\nabla\|\nabla f\|^{2}, \nabla f\right)}{(1-f)^{2}}+\frac{2\left\|\nabla^{2} f\right\|^{2}}{(1-f)^{2}}+\frac{2 \mathcal{R}(\nabla f)}{(1-f)^{2}}-\frac{\Delta\|\nabla f\|^{2}}{(1-f)^{2}}-\frac{2\|\nabla f\|^{2} \Delta f}{(1-f)^{3}} \\
& =-2 g(\nabla w, \nabla f)+2(1-f) w^{2}+\frac{2\left\|\nabla^{2} f\right\|^{2}}{(1-f)^{2}}+\frac{2 \mathcal{R}(\nabla f)}{(1-f)^{2}}-\frac{\Delta\|\nabla f\|^{2}}{(1-f)^{2}}-\frac{2\|\nabla f\|^{2} \Delta f}{(1-f)^{3}} .
\end{aligned}
$$

Combining (4.6) and (4.7), we obtain

$$
\left(\Delta+\partial_{\tau}\right) w-\frac{2 f g(\nabla w, \nabla f)}{1-f}=2(1-f) w^{2}+\frac{2 \mathcal{R}(\nabla f)}{(1-f)^{2}}+2 \mathcal{F},
$$

where

$$
\mathcal{F}:=\frac{\|\nabla f\|^{4}}{(1-f)^{4}}+\frac{\left\|\nabla^{2} f\right\|^{2}}{(1-f)^{2}}+\frac{g\left(\nabla\|\nabla f\|^{2}, \nabla f\right)}{(1-f)^{3}} .
$$

Now, it is enough to verify that $\mathcal{F}$ is non-negative. For the first two terms of (4.8),

$$
\frac{\|\nabla f\|^{4}}{(1-f)^{4}}+\frac{\left\|\nabla^{2} f\right\|^{2}}{(1-f)^{2}} \geq \frac{2\left\|\nabla^{2} f\right\|\|\nabla f\|^{2}}{(1-f)^{3}} \geq \frac{\|\nabla\| \nabla f\left\|^{2}\right\|\|\nabla f\|}{(1-f)^{3}}
$$

by the inequality of arithmetic-geometric means, and the Kato inequality. For the third term, the Cauchy-Schwarz inequality yields

$$
\frac{g\left(\nabla\|\nabla f\|^{2}, \nabla f\right)}{(1-f)^{3}} \geq-\frac{\|\nabla\| \nabla f\left\|^{2}\right\|\|\nabla f\|}{(1-f)^{3}} .
$$

From (4.9) and (4.10), it follows that $\mathcal{F}$ is non-negative, and we complete the proof.

4.2. Cut-off arguments. This subsection is devoted to the cut-off argument. We recall the following elementary fact:

Lemma 4.3. Let $R, T>0, \alpha \in(0,1)$. Then there is a smooth function $\psi:[0, \infty) \times[0, \infty) \rightarrow$ $[0,1]$, and a constant $C_{\alpha}>0$ depending only on $\alpha$ such that the following hold:

(1) $\psi \equiv 1$ on $[0, R / 2] \times[0, T / 4]$, and $\psi \equiv 0$ on $[R, \infty) \times[T / 2, \infty)$;

(2) $\partial_{r} \psi \leq 0$ on $[0, \infty) \times[0, \infty)$, and $\partial_{r} \psi \equiv 0$ on $[0, R / 2] \times[0, \infty)$; 
(3) we have

$$
\frac{\left|\partial_{r} \psi\right|}{\psi^{\alpha}} \leq \frac{C_{\alpha}}{R}, \quad \frac{\left|\partial_{r}^{2} \psi\right|}{\psi^{\alpha}} \leq \frac{C_{\alpha}}{R^{2}}, \quad \frac{\left|\partial_{\tau} \psi\right|}{\psi^{1 / 2}} \leq \frac{C}{T}
$$

where $C>0$ is a universal constant.

Having Lemma 4.3 at hand, we obtain the following assertion:

Proposition 4.4. Let $K \geq 0$. We assume

$$
\mathcal{R}(V) \geq-K\|V\|^{2}, \quad \mathcal{D}(V) \geq-2 K\left(H+\|V\|^{2}\right), \quad \mathcal{H}(V) \geq-\frac{H}{\tau}, \quad H \geq 0
$$

for all vector fields $V$. Let $u: M \times[0, \infty) \rightarrow(0, \infty)$ denote a positive solution to backward heat equation. For $R, T>0$, we assume $u \leq 1$ on $Q_{R, T}$. We define $f$ and $w$ as (4.1) and (4.4) on $Q_{R, T}$, respectively. We also take a function $\psi:[0, \infty) \times[0, \infty) \rightarrow[0,1]$ in Lemma 4.3 with $\alpha=3 / 4$, and define

$$
\psi(x, \tau):=\psi(\mathfrak{d}(x, \tau), \tau)
$$

Then we have

$$
(\psi w)^{2} \leq \frac{\bar{C}_{n}}{R^{4}}+\frac{\widetilde{C}_{1}}{T^{2}}+\widetilde{C}_{2} K^{2}+2 \Phi
$$

at every point in $Q_{R, T}$ such that the reduced distance is smooth, where for the universal constants $C_{3 / 4}, C>0$ given in Lemma 4.3, we put

$$
\begin{aligned}
\bar{C}_{n} & :=24 C_{3 / 4}^{2}\left(n^{2}+\frac{9}{4}+\frac{657}{64} C_{3 / 4}^{2}\right), \quad \widetilde{C}_{1}:=6 C^{2}, \quad \widetilde{C}_{2}:=24\left(1+\frac{C_{3 / 4}^{2}}{4}\right), \\
\Phi & :=\left(\Delta+\partial_{\tau}\right)(\psi w)-\frac{2 g(\nabla \psi, \nabla(\psi w))}{\psi}-\frac{2 f g(\nabla(\psi w), \nabla f)}{1-f} .
\end{aligned}
$$

Proof. First, note that $u \leq 1$ implies $f \leq 0$, and hence $w$ is well-defined. By direct computations and Lemma 4.2,

$$
\begin{aligned}
\Phi & =\psi\left(\Delta+\partial_{\tau}\right) w-\frac{2 \psi f g(\nabla w, \nabla f)}{1-f}+w\left(\Delta+\partial_{\tau}\right) \psi-\frac{2 w\|\nabla \psi\|^{2}}{\psi}-\frac{2 w f g(\nabla \psi, \nabla f)}{1-f} \\
& \geq 2(1-f) \psi w^{2}+\frac{2 \psi \mathcal{R}(\nabla f)}{(1-f)^{2}}+w\left(\Delta+\partial_{\tau}\right) \psi-\frac{2 w\|\nabla \psi\|^{2}}{\psi}-\frac{2 w f g(\nabla \psi, \nabla f)}{1-f} .
\end{aligned}
$$

Therefore,

$$
2(1-f) \psi w^{2} \leq \Psi_{1}+\Psi_{2}+\Psi_{3}+\Psi_{4}+\Phi
$$

for

$$
\Psi_{1}:=-\frac{2 \psi \mathcal{R}(\nabla f)}{(1-f)^{2}}, \quad \Psi_{2}:=-w\left(\Delta+\partial_{\tau}\right) \psi, \quad \Psi_{3}:=\frac{2 w\|\nabla \psi\|^{2}}{\psi}, \quad \Psi_{4}:=\frac{2 w f g(\nabla \psi, \nabla f)}{1-f} .
$$

We present upper estimates of $\Psi_{1}, \Psi_{2}, \Psi_{3}, \Psi_{4}$. The following Young inequality plays an essential role in the estimates: For all $p, q \in(1, \infty)$ with $p^{-1}+q^{-1}=1, a, b \geq 0$, and $\varepsilon>0$,

$$
a b \leq \frac{\varepsilon a^{p}}{p}+\frac{b^{q}}{\varepsilon^{q / p} q} .
$$

The inequality

$$
\frac{\|\nabla \psi\|^{2}}{\psi^{3 / 2}} \leq \frac{3 C_{3 / 4}^{2}}{R^{2}}
$$


is also useful, which follows from Lemmas 3.6 and 4.3. We first derive an upper bound of $\Psi_{1}$. By the assumption for $\mathcal{R}(V)$, the Young inequality (4.18) with $p, q=2$, and $\psi \leq 1$,

$$
\Psi_{1}=-\frac{2 \psi \mathcal{R}(\nabla f)}{(1-f)^{2}} \leq 2 K \psi w \leq \varepsilon \psi^{2} w^{2}+\frac{K^{2}}{\varepsilon} \leq \varepsilon \psi w^{2}+\frac{K^{2}}{\varepsilon}
$$

We next show an upper bound of $\Psi_{2}$. By direct computations, we have

$$
\begin{aligned}
\Psi_{2} & =-w\left(\Delta+\partial_{\tau}\right) \psi=-w\left(\partial_{r} \psi\left(\Delta+\partial_{\tau}\right) \mathfrak{d}+\partial_{r}^{2} \psi\|\nabla \mathfrak{d}\|^{2}+\partial_{\tau} \psi\right) \\
& =-w\left[\partial_{r} \psi\left(\frac{1}{2 \mathfrak{d}}\left(\Delta+\partial_{\tau}\right) \bar{L}-\frac{\|\nabla \bar{L}\|^{2}}{4 \mathfrak{d}^{3}}\right)+\partial_{r}^{2} \psi\|\nabla \mathfrak{d}\|^{2}+\partial_{\tau} \psi\right] \\
& =\frac{w\left|\partial_{r} \psi\right|}{2 \mathfrak{d}}\left(\Delta+\partial_{\tau}\right) \bar{L}-w\left|\partial_{r} \psi\right| \frac{\|\nabla \bar{L}\|^{2}}{4 \mathfrak{d}^{3}}-w \partial_{r}^{2} \psi\|\nabla \mathfrak{d}\|^{2}-w \partial_{\tau} \psi \\
& \leq \frac{w\left|\partial_{r} \psi\right|}{2 \mathfrak{d}}\left(\Delta+\partial_{\tau}\right) \bar{L}+w\left|\partial_{r}^{2} \psi\right|\|\nabla \mathfrak{d}\|^{2}+w\left|\partial_{\tau} \psi\right| .
\end{aligned}
$$

Using Lemmas 3.5, 3.6 and $\bar{L}=\mathfrak{d}^{2}$, we obtain

$$
\begin{aligned}
\Psi_{2} & \leq n \frac{w\left|\partial_{r} \psi\right|}{\mathfrak{d}}+K w\left|\partial_{r} \psi\right| \mathfrak{d}+3 w\left|\partial_{r}^{2} \psi\right|+w\left|\partial_{\tau} \psi\right| \\
& \leq \frac{2 n}{R} w\left|\partial_{r} \psi\right|+K R w\left|\partial_{r} \psi\right|+3 w\left|\partial_{r}^{2} \psi\right|+w\left|\partial_{\tau} \psi\right|,
\end{aligned}
$$

where in the second inequality, we used the fact that $\partial_{r} \psi$ vanishes on $[0, R / 2] \times[0, \infty)$. By the Young inequality (4.18) with $p, q=2$, Lemma 4.3, and $\psi \leq 1$, it holds that

$$
\begin{aligned}
\Psi_{2} \leq\left(\varepsilon \psi w^{2}+\frac{n^{2}}{R^{2}} \frac{\left|\partial_{r} \psi\right|^{2}}{\varepsilon \psi}\right) & +\left(\varepsilon \psi w^{2}+\frac{K^{2} R^{2}}{4} \frac{\left|\partial_{r} \psi\right|^{2}}{\varepsilon \psi}\right) \\
& +\left(\varepsilon \psi w^{2}+\frac{9}{4} \frac{\left|\partial_{r}^{2} \psi\right|^{2}}{\varepsilon \psi}\right)+\left(\varepsilon \psi w^{2}+\frac{1}{4} \frac{\left|\partial_{\tau} \psi\right|^{2}}{\varepsilon \psi}\right) \\
\leq & 4 \varepsilon \psi w^{2}+\frac{C_{3 / 4}^{2}}{\varepsilon}\left(n^{2}+\frac{9}{4}\right) \frac{\psi^{1 / 2}}{R^{4}}+\frac{C^{2}}{4 \varepsilon} \frac{1}{T^{2}}+\frac{C_{3 / 4}^{2}}{4 \varepsilon} K^{2} \psi^{1 / 2} \\
\leq & 4 \varepsilon \psi w^{2}+\frac{C_{3 / 4}^{2}}{\varepsilon}\left(n^{2}+\frac{9}{4}\right) \frac{1}{R^{4}}+\frac{C^{2}}{4 \varepsilon} \frac{1}{T^{2}}+\frac{C_{3 / 4}^{2}}{4 \varepsilon} K^{2} .
\end{aligned}
$$

We next examine an upper bound of $\Psi_{3}$. From the Young inequality (4.18) with $p, q=2$, and (4.19), we deduce

$$
\Psi_{3}=\frac{2 w\|\nabla \psi\|^{2}}{\psi} \leq \varepsilon \psi w^{2}+\frac{\|\nabla \psi\|^{4}}{\varepsilon \psi^{3}} \leq \varepsilon \psi w^{2}+\frac{9 C_{3 / 4}^{4}}{\varepsilon} \frac{1}{R^{4}}
$$

We finally investigate $\Psi_{4}$. The Cauchy-Schwarz inequality, the Young inequality (4.18) with $p=4 / 3, q=4, \varepsilon=4 / 3$, and (4.19) tell us that

$$
\begin{aligned}
\Psi_{4} & =\frac{2 w f g(\nabla \psi, \nabla f)}{1-f} \leq \frac{2 w|f|\|\nabla \psi\|\|\nabla f\|}{1-f}=2 w^{3 / 2}|f|\|\nabla \psi\| \\
& \leq(1-f) \psi w^{2}+\frac{27}{16} \frac{f^{4}}{(1-f)^{3}} \frac{\|\nabla \psi\|^{4}}{\psi^{3}} \leq(1-f) \psi w^{2}+\frac{243 C_{3 / 4}^{4}}{16} \frac{f^{4}}{(1-f)^{3}} \frac{1}{R^{4}}
\end{aligned}
$$


Combining (4.20), (4.24), (4.26), (4.27) with (4.16), we conclude

$$
\begin{aligned}
(1-f) \psi w^{2} \leq 6 \varepsilon \psi w^{2} & +\frac{C_{3 / 4}^{2}}{\varepsilon}\left(n^{2}+\frac{9}{4}+9 C_{3 / 4}^{2}+\frac{243 \varepsilon C_{3 / 4}^{2}}{16} \frac{f^{4}}{(1-f)^{3}}\right) \frac{1}{R^{4}}+\frac{C^{2}}{4 \varepsilon} \frac{1}{T^{2}} \\
& +\frac{1}{\varepsilon}\left(1+\frac{C_{3 / 4}^{2}}{4}\right) K^{2}+\Phi .
\end{aligned}
$$

We divide the both sides by $1-f$. Since $1 /(1-f) \leq 1$ and $f /(1-f) \leq 1$, we see

$$
(1-6 \varepsilon) \psi w^{2} \leq \frac{C_{3 / 4}^{2}}{\varepsilon}\left(n^{2}+\frac{9}{4}+9 C_{3 / 4}^{2}+\frac{243 \varepsilon C_{3 / 4}^{2}}{16}\right) \frac{1}{R^{4}}+\frac{C^{2}}{4 \varepsilon} \frac{1}{T^{2}}+\frac{1}{\varepsilon}\left(1+\frac{C_{3 / 4}^{2}}{4}\right) K^{2}+\Phi .
$$

By letting $\varepsilon \rightarrow 1 / 12$, we obtain

$$
\psi w^{2} \leq \frac{\bar{C}_{n}}{R^{4}}+\frac{\widetilde{C}_{1}}{T^{2}}+\widetilde{C}_{2} K^{2}+2 \Phi
$$

Since $(\psi w)^{2} \leq \psi w^{2}$, we arrive at the desired one (4.13).

4.3. Proof of Theorems $\mathbf{2 . 2}$ and 2.8. We are now in a position to prove Theorem 2.8,

Proof of Theorem 2.8. For $K \geq 0$, let $(M, g(\tau))_{\tau \in[0, \infty)}$ be backward $(-K)$-super Ricci flow satisfying (2.10) for all vector fields $V$, and let $u: M \times[0, \infty) \rightarrow(0, \infty)$ denote a positive solution to backward heat equation. As noticed in [33], it suffices to show the desired estimate for $A=1$. For $R, T>0$, we assume $u \leq 1$ on $Q_{R, T}$. Define functions $f, w$ and $\psi$ as (4.1), (4.4) and (4.12), respectively. For $\theta>0$ we define a compact subset $Q_{R, T, \theta}$ of $Q_{R, T}$ by

$$
Q_{R, T, \theta}:=\left\{(x, \tau) \in Q_{R, T} \mid \tau \in[\theta, T]\right\} .
$$

Hereafter, we fix a small $\theta \in(0, T / 4)$. Also, we take a maximum point $(\bar{x}, \bar{\tau})$ of $\psi w$ in $Q_{R, T, \theta}$.

We first discuss the case where the reduced distance is smooth at $(\bar{x}, \bar{\tau})$. Due to Proposition 4.4, we obtain

$$
(\psi w)^{2} \leq c_{n}\left(\frac{1}{R^{4}}+\frac{1}{T^{2}}+K^{2}\right)+2 \Phi
$$

at $(\bar{x}, \bar{\tau})$ for

$$
c_{n}:=\max \left\{\bar{C}_{n}, \widetilde{C}_{1}, \widetilde{C}_{2}\right\},
$$

where $\bar{C}_{n}, \widetilde{C}_{1}, \widetilde{C}_{2}>0$ and $\Phi$ are defined as (4.14) and (4.15), respectively. Notice that the assumption for $\mathcal{R}(V)$ in (4.11) is satisfied in virtue of the backward $(-K)$-super Ricci flow inequality (2.9) . On the other hand, since $(\bar{x}, \bar{\tau})$ is a maximum point, it holds that

$$
\Delta(\psi w) \leq 0, \quad \partial_{\tau}(\psi w) \leq 0, \quad \nabla(\psi w)=0
$$

at $(\bar{x}, \bar{\tau})$; in particular, $\Phi(\bar{x}, \bar{\tau}) \leq 0$. Therefore, (4.28) implies

$$
(\psi w)(x, \tau) \leq(\psi w)(\bar{x}, \bar{\tau}) \leq c_{n}^{1 / 2}\left(\frac{1}{R^{4}}+\frac{1}{T^{2}}+K^{2}\right)^{1 / 2} \leq c_{n}^{1 / 2}\left(\frac{1}{R^{2}}+\frac{1}{T}+K\right)
$$

for all $(x, \tau) \in Q_{R, T, \theta}$.

We observe the non-smooth case. We employ the barrier argument stated in Remark 3.3 . By the same method as in the proof of Lemma 5.3 in [31], there are a sufficiently small $\delta>0$, a small open neighborhood $U$ of $\bar{x}$ in $M$, and a smooth upper barrier function $\hat{\ell}$ of the reduced distance $\ell$ at $(\bar{x}, \bar{\tau})$ on $U \times(\bar{\tau}-\delta, \bar{\tau}+\delta)$ such that $\widehat{\ell}$ satisfies (3.2), (3.3), (3.4) at $(\bar{x}, \bar{\tau})$. As analogues of $\mathfrak{d}(x, \tau)$ and $\psi(x, \tau)$, we define

$$
\widehat{\mathfrak{d}}(x, \tau):=\sqrt{4 \tau \widehat{\ell}(x, \tau)}, \quad \widehat{\psi}(x, \tau):=\psi(\widehat{\mathfrak{d}}(x, \tau), \tau)
$$


where $\psi$ is a function in Lemma 4.3 with $\alpha=3 / 4$. Remark that $\widehat{\psi}$ is a smooth lower barrier of $\widehat{\psi}$ at $(\bar{x}, \bar{\tau})$ (i.e., $\widehat{\psi} \leq \psi$, and the equality holds at $(\bar{x}, \bar{\tau})$ ); in particular, $(\bar{x}, \bar{\tau})$ is a maximum point of $\widehat{\psi} w$ on $(U \times(\bar{\tau}-\delta, \bar{\tau}+\delta)) \cap Q_{R, T, \theta}$. It follows that

$$
\Delta(\widehat{\psi} w) \leq 0, \quad \partial_{\tau}(\widehat{\psi} w) \leq 0, \quad \nabla(\widehat{\psi} w)=0
$$

at $(\bar{x}, \bar{\tau})$. Having (4.29) at hand, by repeating the calculations in Lemmas 3.4, 3.5, 3.6, and Proposition 4.4, we can verify

$$
(\psi w)(x, \tau) \leq(\psi w)(\bar{x}, \bar{\tau})=(\widehat{\psi} w)(\bar{x}, \bar{\tau}) \leq c_{n}^{1 / 2}\left(\frac{1}{R^{2}}+\frac{1}{T}+K\right)
$$

for all $(x, \tau) \in Q_{R, T, \theta}$. This is the same conclusion as in the smooth case.

In both cases, by $\psi \equiv 1$ on $Q_{R / 2, T / 4, \theta}$, and by the definition of $w$ and $f$,

$$
\frac{\|\nabla u\|}{u} \leq c_{n}^{1 / 4}\left(\frac{1}{R}+\frac{1}{\sqrt{T}}+\sqrt{K}\right)\left(1+\log \frac{1}{u}\right)
$$

on $Q_{R / 2, T / 4, \theta}$. Thus, by letting $\theta \rightarrow 0$, we complete the proof of Theorem 2.8 .

Let us conclude Theorem 2.2 .

Proof of Theorem 2.2. Let $(M, g(\tau))_{\tau \in[0, \infty)}$ be backward super Ricci flow satisfying (2.3) for all vector fields $V$. In each statement, it is enough to show that the gradient of $u$ vanishes at each point in $M \times(0, \infty)$. We fix $(x, \tau) \in M \times(0, \infty)$.

Let us show the first statement. Let $u: M \times[0, \infty) \rightarrow(0, \infty)$ denote a positive solution to backward heat equation. For $R>0$ we put

$$
A_{R}:=\sup _{Q_{R, R^{2}}} u \text {. }
$$

The growth condition (2.4) says $\log \left(A_{R}+1\right)=o(R)$ as $R \rightarrow \infty$. We see $(x, \tau) \in Q_{R / 2, R^{2} / 4}$ for every sufficiently large $R>0$, and fix such one. Applying Theorem 2.8 with $K=0$ to a function $u+1$ on $Q_{R, R^{2}}$ leads us to

$$
\frac{\|\nabla u\|}{u+1} \leq \frac{2 C_{n}}{R}\left(1+\log \frac{A_{R}+1}{u+1}\right) \leq \frac{2 C_{n}}{R}\left(1+\log \left(A_{R}+1\right)\right)=\frac{2 C_{n}}{R}(1+o(R))
$$

at $(x, \tau)$. Letting $R \rightarrow \infty$, we arrive at the desired conclusion.

We next prove the second statement. Let $u: M \times[0, \infty) \rightarrow \mathbb{R}$ be a solution to backward heat equation. For $R>0$ we set

$$
\bar{A}_{R}:=\sup _{Q_{R, R^{2}}}|u| .
$$

The growth condition (2.5) tells us $\bar{A}_{R}=o(R)$ as $R \rightarrow \infty$. We now fix a sufficiently large $R>0$ such that $(x, \tau) \in Q_{R / 2, R^{2} / 4}$, and apply Theorem 2.8 to $u+2 \bar{A}_{R}$ on $Q_{R, R^{2}}$. Since

$$
\bar{A}_{R} \leq u+2 \bar{A}_{R} \leq 3 \bar{A}_{R}
$$

on $Q_{R, R^{2}}$, we have

$$
\|\nabla u\| \leq \frac{2 C_{n}}{R}\left(1+\log \frac{3 \bar{A}_{R}}{u+2 \bar{A}_{R}}\right)\left(u+2 \bar{A}_{R}\right) \leq 6(1+\log 3) C_{n} \frac{o(R)}{R}
$$

at $(x, \tau)$. By letting $R \rightarrow \infty$, we complete the proof of Theorem 2.2 .

\section{Trace Harnack inequalities and gradient estimates for $K$-Ricci flow}

In this section, we prove Corollary 2.10. The proof is done by extending the Hamilton's trace Harnack inequality for Ricci flow to $K$-Ricci flow. 
5.1. Trace Harnack inequalities. In the present subsection, we study the trace Harnack inequality for $K$-Ricci flow. To do so, we prepare several key tools. For $K \neq 0$, we define a monotone increasing, bijective function $\sigma: J \rightarrow \mathbb{R}$ by

$$
\sigma(s):=-\frac{1}{2 K} \log (1-2 K s)
$$

where

$$
J:= \begin{cases}(-\infty, 1 / 2 K) & \text { if } K>0 \\ (1 / 2 K, \infty) & \text { if } K<0\end{cases}
$$

This function satisfies

$$
\sigma^{\prime}(s)=e^{2 K \sigma(s)} .
$$

Furthermore, its inverse function $\sigma^{-1}: \mathbb{R} \rightarrow J$ is given by

$$
\sigma^{-1}(t)=\frac{1-e^{-2 K t}}{2 K}
$$

with $\sigma^{-1}(0)=0$. Our proof is based on the following observation (cf. Theorem 1.11 in [24]):

Lemma 5.1. Let $K \neq 0$, and let $(M, g(t))_{t \in[0, \mathcal{T})}$ be a time-dependent Riemannian manifold. For $s \in\left[0, \sigma^{-1}(\mathcal{T})\right)$ we define

$$
\bar{g}(s):=e^{-2 K \sigma(s)} g(\sigma(s)) .
$$

Then the following are equivalent:

(1) $(M, g(t))_{t \in[0, \mathcal{T})}$ is K-Ricci flow;

(2) $(M, \bar{g}(s))_{s \in\left[0, \sigma^{-1}(\mathcal{T})\right)}$ is Ricci flow.

Proof. The property (5.1) yields

$$
\partial_{s} \bar{g}=\sigma^{\prime}(s) e^{-2 K \sigma(s)}\left(-2 K g+\partial_{t} g\right)=-2 K g+\partial_{t} g .
$$

We arrive at the desired assertion since the Ricci curvature is invariant under multiplication of Riemannian metric by positive constants.

The following is the main result of this subsection:

Theorem 5.2 ([18]). For $K \in \mathbb{R}$, let $(M, g(t))_{t \in[0, \mathcal{T})}$ denote a complete $K$-Ricci flow with bounded, non-negative curvature operator. Then we have

$$
\partial_{t} S+\frac{2 K S}{1-e^{-2 K t}}-2 g(\nabla S, V)+2 \operatorname{Ric}(V, V) \geq 0
$$

for all vector fields $V$ and $t>0$, where $S$ denotes the scalar curvature. In the case of $K=0$, we interpret $2 K /\left(1-e^{-2 K t}\right)$ in the second term as the limit $1 / t$.

Proof. When $K=0$, this theorem is nothing but the Hamilton's trace Harnack inequality for Ricci flow (see Corollary 1.2 in [18]). We now investigate the case of $K \neq 0$. By virtue of Lemma 5.1, we can construct an associated Ricci flow $(M, \bar{g}(s))_{s \in\left[0, \sigma^{-1}(\mathcal{T})\right)}$ determined by (5.3), which also has bounded, non-negative curvature operator. The idea is to apply the Hamilton's trace Harnack inequality to this Ricci flow. By doing that,

$$
\partial_{s} \bar{S}+\frac{\bar{S}}{s}-2 \bar{g}(\bar{\nabla} \bar{S}, \bar{V})+2 \overline{\operatorname{Ric}}(\bar{V}, \bar{V}) \geq 0
$$


for all vector fields $\bar{V}$ and $s>0$, where $\bar{\nabla}, \overline{\operatorname{Ric}}, \bar{S}$ are the gradient, Ricci curvature, scalar curvature induced from $\bar{g}(s)$, respectively. Let us translate (5.4) into the language of $g(t)$. One can verify

$$
\begin{aligned}
\overline{\operatorname{Ric}}(\bar{V}, \bar{V}) & =\operatorname{Ric}(\bar{V}, \bar{V}), \quad \bar{S}=e^{2 K \sigma(s)} S, \quad \bar{g}(\bar{\nabla} \bar{S}, \bar{V})=e^{2 K \sigma(s)} g(\nabla S, \bar{V}), \\
\partial_{s} \bar{S} & =\sigma^{\prime}(s) e^{2 K \sigma(s)}\left(2 K S+\partial_{t} S\right)=e^{4 K \sigma(s)}\left(2 K S+\partial_{t} S\right) .
\end{aligned}
$$

Substituting these equations into (5.4), we obtain

$$
e^{4 K t}\left(2 K S+\partial_{t} S\right)+\frac{e^{2 K t} S}{\sigma^{-1}(t)}-2 e^{2 K t} g(\nabla S, \bar{V})+2 \operatorname{Ric}(\bar{V}, \bar{V}) \geq 0
$$

for all vector fields $\bar{V}$ and $t>0$. In particular, (5.2) leads to

$$
\partial_{t} S+\frac{2 K S}{1-e^{-2 K t}}-2 g\left(\nabla S, e^{-2 K t} \bar{V}\right)+2 \operatorname{Ric}\left(e^{-2 K t} \bar{V}, e^{-2 K t} \bar{V}\right) \geq 0 .
$$

For a given vector field $V$, we complete the proof by applying (5.5) to $\bar{V}:=e^{2 K t} V$.

We can derive the following trace Harnack inequality for ancient $K$-Ricci flow:

Corollary $5.3([18])$. For $K \in \mathbb{R}$, let $(M, g(t))_{t \in(-\infty, 0]}$ be a complete ancient $K$-Ricci flow with bounded, non-negative curvature operator. Then for all vector fields $V$, we have:

(1) If $K>0$, then

$$
\partial_{t} S+2 K S-2 g(\nabla S, V)+2 \operatorname{Ric}(V, V) \geq 0
$$

(2) if $K \leq 0$, then

$$
\partial_{t} S-2 g(\nabla S, V)+2 \operatorname{Ric}(V, V) \geq 0 .
$$

Proof. The desired inequalities follow from the standard argument. For a negative $t_{0}<0$, by applying Theorem 5.2 to a parallel translation $(M, \widetilde{g}(s))_{s \in\left[0,-t_{0}\right)}$ defined as $\widetilde{g}(s):=g\left(s+t_{0}\right)$, we see that

$$
\partial_{t} S+\frac{2 K S}{1-e^{-2 K\left(t-t_{0}\right)}}-2 g(\nabla S, V)+2 \operatorname{Ric}(V, V) \geq 0
$$

for all vector fields $V$ and $t \in\left(t_{0}, 0\right)$. Now, for a given time $t \in(-\infty, 0)$, we take $t_{0}<0$ such that $t_{0}<t$. Letting $t_{0} \rightarrow-\infty$ in (5.7), we arrive at the desired claim.

5.2. Proof of Corollary 2.10. The aim of this subsection is to give a proof of Corollary 2.10. Besides Corollary 5.3, we will use the following:

Lemma 5.4. For $K \geq 0$, let $(M, g(\tau))_{\tau \in[0, \infty)}$ be a backward $(-K)$-Ricci flow. Then for all vector fields $V$, we have

$$
\mathcal{D}(V)=-2 K\left(H+\|V\|^{2}\right) .
$$

Proof. The backward $(-K)$-Ricci flow equation can be written as

$$
h=\operatorname{Ric}+K g \text {; }
$$

in particular, for $\mathcal{R}(V)$ defined by (2.7),

$$
\mathcal{R}(V)=-K\|V\|^{2} .
$$

On the other hand, by taking the trace of (5.8), we see

$$
H=S+n K
$$

for the scalar curvature $S$, where $n$ denotes the dimension of $M$. Let us substitute (5.10) into the definition of $\mathcal{D}_{0}(V)$ defined as (2.6) . By the contracted second Bianchi identity,

$$
\mathcal{D}_{0}(V)=-\partial_{\tau} S-\Delta S-2\|\operatorname{Ric}\|^{2}-4 K S-2 n K^{2} .
$$


Also, we possess the following evolution formula for $S$ (see e.g., Proposition 4.10 in [1]):

$$
\partial_{\tau} S=-\Delta S-2\|\operatorname{Ric}\|^{2}-2 K S .
$$

Combining (5.11) and (5.12), we obtain

$$
\mathcal{D}_{0}(V)=-2 K S-2 n K^{2}=-2 K H .
$$

From (5.9) and (5.13), we conclude the desired formula.

We are now in a position to prove Corollary 2.10.

Proof of Corollary 2.10. For $K \geq 0$, let $(M, g(\tau))_{\tau \in[0, \infty)}$ be an $n$-dimensional, complete backward $(-K)$-Ricci flow with bounded, non-negative curvature operator. It suffices to check that $(M, g(\tau))_{\tau \in[0, \infty)}$ satisfies the assumption in Theorem 2.8.

By the backward $(-K)$-Ricci flow equation, for the scalar curvature $S$, we have

$$
h=\mathrm{Ric}+K g, \quad H=S+n K .
$$

In particular, by the non-negativity of curvature operator, the admissibility and the nonnegativity of $H$ in (2.10) are satisfied. Lemma 5.4 yields the assumption for $\mathcal{D}(V)$ in (2.10). Due to (5.6) in Corollary 5.3 and $K \geq 0$,

$$
\mathcal{H}(V)+\frac{H}{\tau}=-\partial_{\tau} S-2 g(\nabla S, V)+2 \operatorname{Ric}(V, V)+2 K\|V\|^{2} \geq 0,
$$

which is the assumption for $\mathcal{H}(V)$ in (2.10). We complete the proof of Corollary 2.10.

\section{Comparisons With other SPACE-Only local Gradient estimates}

In this section, we compare Theorem 2.8 with other space-only local gradient estimates. We aim to make our contribution clear.

6.1. Souplet-Zhang gradient estimate. We first compare our method of the proof with that of Souplet-Zhang gradient estimate in 33 .

As stated in Section 4, we have proved Theorem 2.8 along the line of the proof of SoupletZhang gradient estimate. On the other hand, there exists only one technically different part. That is the upper estimate of $\Psi_{2}$ demonstrated in (4.21), (4.23) and (4.24).

In [33], they have obtained such an upper estimate by giving upper bounds of

$$
\left(\Delta+\partial_{\tau}\right) \mathfrak{d}
$$

and

$$
\|\nabla \mathfrak{d}\|^{2}
$$

at the stage of (4.21). In the static case, $\mathfrak{d}$ coincides with the Riemannian distance function from the fixed point (see Remark 2.3). Then they have estimated (6.1) by use of the Laplacian comparison in Riemannian geometry, and the fact that its time derivative vanishes. For (6.2), they did nothing in fact since it is identical to a universal constant 1.

In our case, instead of (6.1), we provide an upper bound of

$$
\left(\Delta+\partial_{\tau}\right) \bar{L}
$$

in (4.22) by using Lemma 3.5, which is a combination of the Laplacian comparison (3.4) in reduced geometry, and the time derivative formula (3.2) for reduced distance. Moreover, we could give a universal upper bound of (6.2) in (4.22) by Lemma 3.6 (cf. Remark 3.7). 
6.2. Bailesteanu-Cao-Pulemotov gradient estimate. We attempt to compare Theorem 2.8 with other space-only local gradient estimates by Bailesteanu-Cao-Pulemotov [3], Zhang [41], and Ecker-Knopf-Ni-Topping [11]. To make it easier, in the next three subsections, we translate their results into our setting and notation.

In this subsection, we focus on the work of Bailesteanu-Cao-Pulemotov [3], which is most closely related to our work. In [3], they have produced a space-only local gradient estimate for positive solutions to heat equation along Ricci flow over positive time interval (see Theorem 2.2 in [3]). In our notation, we can show the following gradient estimate of Bailesteanu-CaoPulemotov type by inserting the key techniques in [3] into the proof of Theorem 2.8.

Theorem $6.1([3])$. Let $(M, g(\tau))_{\tau \in[0, \infty)}$ be an n-dimensional, complete backward super Ricci flow. For $K \geq 0$, we assume

$$
\mid \text { Ric } \mid \leq K g \text {. }
$$

Let $u: M \times[0, \infty) \rightarrow(0, \infty)$ be a positive solution to backward heat equation. For $R, T>0$ and $A>0$, we suppose $u \leq A$ on

$$
B_{R, T}:=\{(x, \tau) \in M \times[0, T] \mid d(x, \tau) \leq R\},
$$

where $d(x, \tau)$ denotes the Riemannian distance from a fixed point induced from $g(\tau)$. Then there exists a positive constant $C_{n}>0$ depending only on $n$ such that on $B_{R / 2, T / 4}$,

$$
\frac{\|\nabla u\|}{u} \leq C_{n}\left(\frac{1}{R}+\frac{1}{\sqrt{T}}+\sqrt{K}\right)\left(1+\log \frac{A}{u}\right) .
$$

Proof. We only sketch the proof. We can prove it only by replacing the role of $\mathfrak{d}(x, \tau)$ with $d(x, \tau)$ in the definition of the cut-off function $\psi$ defined as (4.12) in Lemma 4.4. For such function $\psi$, we need to present associated upper estimates for $\Psi_{1}, \Psi_{2}, \Psi_{3}, \Psi_{4}$ defined as (4.17).

First, $\Psi_{1}$ must be non-positive in virtue of the backward super Ricci flow inequality (2.2). We discuss an upper estimate of $\Psi_{2}$. Here we need the key techniques in [3]. Since the Ricci curvature is bounded from below in (6.3), the Laplacian comparison implies

$$
\Delta d \leq(n-1) \sqrt{\frac{K}{n-1}} \frac{\cosh \sqrt{(n-1)^{-1} K} d}{\sinh \sqrt{(n-1)^{-1} K} d} \leq(n-1)\left(\frac{1}{d}+\sqrt{\frac{K}{n-1}}\right) .
$$

Furthermore, the backward super Ricci flow inequality (2.2) and the upper Ricci curvature bound in (6.3) lead us to

$$
\partial_{\tau} d=\int_{0}^{d} h\left(\gamma^{\prime}(s), \gamma^{\prime}(s)\right) d s \leq \int_{0}^{d} \operatorname{Ric}\left(\gamma^{\prime}(s), \gamma^{\prime}(s)\right) d s \leq K d
$$

for a unit speed minimal geodesic $\gamma:[0, d] \rightarrow M$ from the fixed point (see e.g., Lemma 18.1 in [9]). We now use (6.4), (6.5) in (4.21) instead of Lemma 3.5] in (4.22), and $\|\nabla d\|=1$ in (4.21) instead of Lemma 3.6 in (4.22), which implies a similar upper estimate to (4.24). Note that due to the second term of the right hand side of (6.4), an additional term regarding $K / R^{2}$ appears in (4.25). We deal with such a term by dividing it into $K^{2}$-part and $1 / R^{4}$-part with the help of the inequality of arithmetic-geometric means (cf. [33]).

We also consider upper estimates of $\Psi_{3}, \Psi_{4}$. In view of $\|\nabla d\|=1$, we possess

$$
\frac{\|\nabla \psi\|^{2}}{\psi^{3 / 2}} \leq \frac{C_{3 / 4}^{2}}{R^{2}}
$$

instead of (4.19). We obtain the desired estimates of $\Psi_{3}, \Psi_{4}$ by using (6.6) in (4.26) and in (4.27) instead of (4.19), respectively. Based on these estimates, we can prove the desired gradient estimate by the same argument as in the proof of Theorem 2.8 . 
Remark 6.2. We can not conclude the associated Liouville theorem from Theorem 6.1 except for a quite specific case. Actually, to do so, we need to apply Theorem 6.1 with $K=0$. But in that case, it must be Ricci flat by the assumption (6.3).

6.3. Zhang gradient estimate. In the present subsection, we are concerned with the work of Zhang [41]. He proved a space-only local gradient estimate for positive solutions to heat equation along backward ancient Ricci flow (see Theorem 3.1 (a) in [41]). We emphasize that he has dealt with not Ricci flow but backward one. In the same spirit as in Subsection 6.2, we can prove the following gradient estimate of Zhang type:

Theorem $6.3([41])$. Let $(M, g(\tau))_{\tau \in[0, \infty)}$ denote an $n$-dimensional, complete sub Ricci flow, namely, a subsolution to the Ricci flow equation (1.1) defined by

$$
\text { Ric } \leq-h
$$

For $K \geq 0$, we assume

$$
\text { Ric } \geq-K g \text {. }
$$

Let $u: M \times[0, \infty) \rightarrow(0, \infty)$ be a positive solution to backward heat equation. For $R, T>0$ and $A>0$, we suppose $u \leq A$ on $B_{R, T}$. Then there exists a positive constant $C_{n}>0$ depending only on $n$ such that on $B_{R / 2, T / 4}$,

$$
\frac{\|\nabla u\|}{u} \leq C_{n}\left(\frac{1}{R}+\frac{1}{\sqrt{T}}+\sqrt{K}\right)\left(1+\log \frac{A}{u}\right) .
$$

Proof. We only outline its proof. In the same manner as Theorem 6.1, one can show it only by considering the cut-off function $\psi$ determined by not $\mathfrak{d}(x, \tau)$ but $d(x, \tau)$ in Lemma 4.4 . We explain the way to bound $\Psi_{1}, \Psi_{2}, \Psi_{3}, \Psi_{4}$ defined as (4.17) in this setting. We stress again that we now deal with not backward Ricci flow but forward one unlike Theorem 6.1, Moreover, we only have a lower Ricci curvature bound (6.8) unlike (6.3).

For $\Psi_{1}$, the sub Ricci flow inequality (6.7), the lower Ricci curvature bound (6.8), the Young inequality (4.18) with $p, q=2$, and $\psi \leq 1$ yield

$$
\Psi_{1}=-\frac{2 \psi \mathcal{R}(\nabla f)}{(1-f)^{2}} \leq-\frac{4 \psi \operatorname{Ric}(\nabla f, \nabla f)}{(1-f)^{2}} \leq 4 K \psi w \leq \varepsilon \psi^{2} w^{2}+\frac{4 K^{2}}{\varepsilon} \leq \varepsilon \psi w^{2}+\frac{4 K^{2}}{\varepsilon}
$$

which corresponds to (4.20). On $\Psi_{2}$, the sub Ricci flow inequality (6.7) and the lower Ricci curvature bound in (6.8) also yield

$$
\partial_{\tau} d=\int_{0}^{d} h\left(\gamma^{\prime}(s), \gamma^{\prime}(s)\right) d s \leq-\int_{0}^{d} \operatorname{Ric}\left(\gamma^{\prime}(s), \gamma^{\prime}(s)\right) d s \leq K d
$$

for a unit speed minimal geodesic $\gamma:[0, d] \rightarrow M$ from the fixed point. Since the conclusion of (6.10) is same as that of (6.5), this together with the Laplacian comparison (6.4) yields the same upper estimate of $\Psi_{2}$ as in Theorem 6.1. Also, we have the same upper estimates of $\Psi_{3}, \Psi_{4}$. Thus, we complete the proof by the same argument as in Theorem 2.8.

Unlike Theorem 6.1, one can conclude the following Liouville theorem by use of Theorem 6.3 with $K=0$ :

Theorem 6.4 ([41]). Let $(M, g(\tau))_{\tau \in[0, \infty)}$ be a complete sub Ricci flow of non-negative Ricci curvature. Then we have the following:

(1) Let $u: M \times[0, \infty) \rightarrow(0, \infty)$ be a positive solution to backward heat equation. If

$$
u(x, \tau)=\exp [o(d(x, \tau)+\sqrt{\tau})]
$$

near infinity, then $u$ is constant; 
(2) let $u: M \times[0, \infty) \rightarrow \mathbb{R}$ be a solution to backward heat equation. If

$$
u(x, \tau)=o(d(x, \tau)+\sqrt{\tau})
$$

near infinity, then $u$ is constant.

6.4. Ecker-Knopf-Ni-Topping gradient estimate. We discuss the work of Ecker-KnopfNi-Topping [11. They have investigated a space-only local gradient estimate for positive solutions to conjugate heat equation along general geometric flow over positive time interval (see Theorem 10 in [11]). Thanks to their method, we can formulate the following gradient estimate of Ecker-Knopf-Ni-Topping in our setting and notation:

Theorem $6.5([11])$. Let $(M, g(\tau))_{\tau \in[0, \infty)}$ be an $n$-dimensional, complete sub Ricci flow. For $K, \bar{K} \geq 0$, we assume

$$
\text { Ric } \geq-K g, \quad\|\nabla H\| \leq \bar{K} .
$$

Let $u: M \times[0, \infty) \rightarrow(0, \infty)$ be a positive solution to conjugate heat equation

$$
\left(\Delta+\partial_{\tau}+H\right) u=0 .
$$

For $R, T>0$ and $A>0$, we suppose $u \leq A$ on $B_{R, T}$. Then there exists a positive constant $C_{n}>0$ depending only on $n$ such that on $B_{R / 2, T / 4}$,

$$
\frac{\|\nabla u\|}{u} \leq C_{n}\left(\frac{1}{R}+\frac{1}{\sqrt{T}}+\sqrt{K}+\sqrt{\bar{K}}+\bar{K}^{1 / 4}\right)\left(1+\log \frac{A}{u}\right) .
$$

Proof. Let us give an outline of the proof. To begin with, we need to prepare alternatives of Lemmas 4.1 and 4.2 for positive solution $u: M \times[0, \infty) \rightarrow(0, \infty)$ to conjugate heat equation (6.12). Under the same setting as in Lemma 4.1, we can verify

$$
\begin{aligned}
\left(\Delta+\partial_{\tau}\right) f & =-\|\nabla f\|^{2}-H, \\
\left(\Delta+\partial_{\tau}\right)\|\nabla f\|^{2} & =2\left\|\nabla^{2} f\right\|^{2}-2 g\left(\nabla\|\nabla f\|^{2}, \nabla f\right)+2 \mathcal{R}(\nabla f)-2 g(\nabla H, \nabla f)
\end{aligned}
$$

by the same calculation as in the proof of Lemma 4.1. Keeping (6.14), (6.15) in mind, under the same setting as in Lemma 4.2, one can also deduce

$$
\left(\Delta+\partial_{\tau}\right) w-\frac{2 f g(\nabla w, \nabla f)}{1-f} \geq 2(1-f) w^{2}+\frac{2 \mathcal{R}(\nabla f)}{(1-f)^{2}}-\frac{2 g(\nabla H, \nabla f)}{(1-f)^{2}}-\frac{2 H\|\nabla f\|^{2}}{(1-f)^{3}}
$$

from the same calculation as in the proof of Lemma 4.2.

Similarly to Theorem [6.3, we consider the cut-off function $\psi$ determined by not $\mathfrak{d}(x, \tau)$ but $d(x, \tau)$ in Lemma 4.4. In virtue of (6.16), it holds that

$$
2(1-f) \psi w^{2} \leq \Psi_{1}+\Psi_{2}+\Psi_{3}+\Psi_{4}+\Psi_{5}+\Psi_{6}+\Phi
$$

for $\Psi_{1}, \Psi_{2}, \Psi_{3}, \Psi_{4}$ defined as (4.17), for $\Psi_{5}, \Psi_{6}$ defined by

$$
\Psi_{5}:=\frac{2 \psi g(\nabla H, \nabla f)}{(1-f)^{2}}, \quad \Psi_{6}:=\frac{2 H \psi\|\nabla f\|^{2}}{(1-f)^{3}},
$$

and for $\Phi$ defined as (4.15). Since we assume the sub Ricci flow inequality (6.7) and the lower Ricci curvature bound in (6.11), one can derive the same upper bounds for $\Psi_{1}, \Psi_{2}, \Psi_{3}, \Psi_{4}$ as in Theorem 6.3. Hence, it suffices to examine upper bounds of $\Psi_{5}, \Psi_{6}$. By the CauchySchwarz inequality, the assumption (6.11) for $\|\nabla H\|$, the facts $1 /(1-f) \leq 1$ and $\psi \leq 1$, the inequality of arithmetic-geometric means, and the Young inequality (4.18) with $p, q=2$,

$$
\begin{aligned}
\Psi_{5} & =\frac{2 \psi g(\nabla H, \nabla f)}{(1-f)^{2}} \leq \frac{2 \psi\|\nabla H\|\|\nabla f\|}{(1-f)^{2}} \leq \frac{2 \bar{K} \psi\|\nabla f\|}{1-f} \\
& \leq 2 \bar{K}(\psi w)^{1 / 2} \leq \bar{K}(1+\psi w) \leq \bar{K}+\varepsilon \psi w^{2}+\frac{1}{4} \frac{\bar{K}^{2}}{\varepsilon},
\end{aligned}
$$


where the first term in the right hand side of the above inequality will be reduced to $\bar{K}^{1 / 4}$ term in the right hand side of (6.13). Combining the sub Ricci flow inequality (6.7) and the lower Ricci curvature bound in (6.11), we possess

$$
h \leq-\mathrm{Ric} \leq K g, \quad H \leq n K,
$$

and hence

$$
\Psi_{6}=\frac{2 H \psi\|\nabla f\|^{2}}{(1-f)^{3}} \leq \frac{2 n K \psi\|\nabla f\|^{2}}{(1-f)^{3}} \leq 2 n K \psi w \leq \varepsilon \psi w^{2}+\frac{n^{2} K^{2}}{\varepsilon} .
$$

Here we used $1 /(1-f) \leq 1$ and the Young inequality (4.18) with $p, q=2$. Summarizing these upper bounds, we arrive at the desired gradient estimate.

Remark 6.6. Similarly to Theorem 6.1, we can not deduce the associated Liouville theorem from Theorem 6.5 except for a quite specific case. For that purpose, we use Theorem 6.1 with $K=0$ and $\bar{K}=0$. Then, however, $H$ must be constant by the assumption (6.11).

6.5. Discussion. Based on the observation in the above three subsections, we now describe our contributions comparing Theorem 2.8 with other space-only local gradient estimates.

Due to the work of Zhang [41, we see that the Liouville theorem of Souplet-Zhang type holds for backward heat equation along sub Ricci flow under a growth condition for Riemannian distance (see Theorem 6.4). Meanwhile, in view of the work of Bailesteanu-CaoPulemotov [3], it seems to be difficult to yield such a Liouville theorem along backward super Ricci flow (see Theorem 6.1 and Remark 6.2). One of our contributions is to point out that we can formulate the Liouville theorem by considering a growth condition with regard to reduced distance instead of Riemannian distance.

The reason why Theorem 6.1 does not imply the associated Liouville theorem is that the upper Ricci curvature bound is assumed, which leads to an upper bound of the time derivative of Riemannian distance (see (6.5)). Note that along sub Ricci flow, such an upper bound is derived from the lower Ricci curvature bound (see $(\underline{6.10})$ ). In the proof of Theorem 2.8, we overcome this issue by using the time derivative formula (3.2) for reduced distance.

In the proof of Theorem 2.8, we need to control not only the time derivative for reduced distance but also its Laplacian. In Theorems 6.1, 6.3, 6.5, the Laplacian of Riemannian distance is controlled by the lower Ricci curvature bound (see (6.4)). On the other hand, in analyzing the Laplacian of reduced distance, the lower Ricci curvature bound seems not to be compatible with our situation. Our another contribution is to provide an insight that the non-negative number $K$ appeared in the lower Ricci curvature bound in (6.3), (6.8), (6.11) should be included in the super Ricci flow inequality (i.e., $(-K)$-super Ricci flow inequality). Then we can complete the proof by the Laplacian comparison (3.4) for reduced distance.

Acknowledgements. The first author was supported by JSPS KAKENHI (JP19K14521). The second author was supported by JSPS Grant-in-Aid for Scientific Research on Innovative Areas "Discrete Geometric Analysis for Materials Design" (17H06460).

\section{REFERENCES}

[1] B. Andrews and C. Hopper, The Ricci flow in Riemannian geometry. A complete proof of the differentiable 1/4-pinching sphere theorem, Lecture Notes in Mathematics, 2011. Springer, Heidelberg, 2011. xviii+296 pp.

[2] M. Arnaudon, K. A. Coulibaly and A. Thalmaier, Horizontal diffusion in $C^{1}$ path space, Séminaire de Probabilités XLIII, 73-94, Lecture Notes in Math., 2006, Springer, Berlin, 2011.

[3] M. Bailesteanu, X. Cao and A. Pulemotov, Gradient estimates for the heat equation under the Ricci flow, J. Funct. Anal. 258 (2010), no. 10, 3517-3542.

[4] X. Cao, Differential Harnack estimates for backward heat equations with potentials under the Ricci flow, J. Funct. Anal. 255 (2008), no. 4, 1024-1038. 
[5] X. Cao, H. Guo and H. Tran, Harnack estimates for conjugate heat kernel on evolving manifolds, Math. Z. 281 (2015), no. 1-2, 201-214.

[6] X. Cao and R. S. Hamilton, Differential Harnack estimates for time-dependent heat equations with potentials, Geom. Funct. Anal. 19 (2009), no. 4, 989-1000.

[7] S. Y. Cheng and S. T. Yau, Differential equations on Riemannian manifolds and their geometric applications, Comm. Pure Appl. Math. 28 (1975), no. 3, 333-354.

[8] B. Chow, S-C. Chu, D. Glickenstein, C. Guenther, J. Isenberg, T. Ivey, D. Knopf, P. Lu, F. Luo and L. $\mathrm{Ni}$, The Ricci flow: techniques and applications. Part I. Geometric aspects, Mathematical Surveys and Monographs, 135. American Mathematical Society, Providence, RI, 2007. xxiv+536 pp.

[9] B. Chow, S-C. Chu, D. Glickenstein, C. Guenther, J. Isenberg, T. Ivey, D. Knopf, P. Lu, F. Luo and L. Ni, The Ricci flow: techniques and applications. Part III. Geometric-analytic aspects, Mathematical Surveys and Monographs, 163. American Mathematical Society, Providence, RI, 2010. xx+517 pp.

[10] T. H. Colding and W. P. Minicozzi II, Liouville properties, ICCM Not. 7 (2019), no. 1, 16-26.

[11] K. Ecker, D. Knopf, L. Ni and P. Topping, Local monotonicity and mean value formulas for evolving Riemannian manifolds, J. Reine Angew. Math. 616 (2008), 89-130.

[12] S. Fang, Differential Harnack inequalities for heat equations with potentials under geometric flows, Arch. Math. (Basel) 100 (2013), no. 2, 179-189.

[13] S. Fang and P. Zhu, Differential Harnack estimates for backward heat equations with potentials under geometric flows, Commun. Pure Appl. Anal. 14 (2015), no. 3, 793-809.

[14] H. Guo and T. He, Harnack estimates for geometric flows, applications to Ricci flow coupled with harmonic map flow, Geom. Dedicata 169 (2014), 411-418.

[15] H. Guo, R. Philipowski and A. Thalmaier, Entropy and lowest eigenvalue on evolving manifolds, Pacific J. Math. 264 (2013), no. 1, 61-81.

[16] H. Guo, R. Philipowski and A. Thalmaier, An entropy formula for the heat equation on manifolds with time-dependent metric, application to ancient solutions, Potential Anal. 42 (2015), no. 2, 483-497.

[17] R. S. Hamilton, Three-manifolds with positive Ricci curvature, J. Differential Geometry 17 (1982), no. 2, 255-306.

[18] R. S. Hamilton, The Harnack estimate for the Ricci flow, J. Differential Geom. 37 (1993), no. 1, $225-243$.

[19] R. S. Hamilton, A matrix Harnack estimate for the heat equation, Comm. Anal. Geom. 1 (1993), no. 1, 113-126.

[20] R. Haslhofer and A. Naber, Characterizations of the Ricci flow, J. Eur. Math. Soc. (JEMS) 20 (2018), no. 5, 1269-1302.

[21] H. Huang, Optimal transportation and monotonic quantities on evolving manifolds, Pacific J. Math. 248 (2010), no. 2, 305-316.

[22] M. Ishida, Geometric flows and differential Harnack estimates for heat equations with potentials, Ann. Global Anal. Geom. 45 (2014), no. 4, 287-302.

[23] E. Kopfer, Super-Ricci flows and improved gradient and transport estimates, Probab. Theory Related Fields 175 (2019), no. 3-4, 897-936.

[24] E. Kopfer and K.-T. Sturm, Heat flow on time-dependent metric measure spaces and super-Ricci flows, Comm. Pure Appl. Math. 71 (2018), no. 12, 2500-2608.

[25] S. Li and X.-D. Li, The $W$-entropy formula for the Witten Laplacian on manifolds with time dependent metrics and potentials, Pacific J. Math. 278 (2015), no. 1, 173-199.

[26] S. Li and X.-D. Li, Hamilton differential Harnack inequality and W-entropy for Witten Laplacian on Riemannian manifolds, J. Funct. Anal. 274 (2018), no. 11, 3263-3290.

[27] S. Li and X.-D. Li, On Harnack inequalities for Witten Laplacian on Riemannian manifolds with super Ricci flows, Asian J. Math. 22 (2018), no. 3, 577-597.

[28] S. Li and X.-D. Li, W-entropy, super Perelman Ricci flows and $(K, m)$-Ricci solitons, to appear in J. Geom. Anal..

[29] J. Lott and C. Villani, Ricci curvature for metric-measure spaces via optimal transport, Ann. of Math. (2) 169 (2009), no. 3, 903-991.

[30] R. J. McCann and P. M. Topping, Ricci flow, entropy and optimal transportation, Amer. J. Math. 132 (2010), no. 3, 711-730.

[31] R. Müller, Monotone volume formulas for geometric flows, J. Reine Angew. Math. 643 (2010), 39-57.

[32] G. Perelman, The entropy formula for the Ricci flow and its geometric applications, preprint arXiv:math/0211159.

[33] P. Souplet and Q. S. Zhang, Sharp gradient estimate and Yau's Liouville theorem for the heat equation on noncompact manifolds, Bull. London Math. Soc. 38 (2006), no. 6, 1045-1053.

[34] K.-T. Sturm, On the geometry of metric measure spaces. I, Acta Math. 196 (2006), no. 1, 65-131. 
[35] K.-T. Sturm, On the geometry of metric measure spaces. II, Acta Math. 196 (2006), no. 1, 133-177.

[36] K.-T. Sturm, Super-Ricci flows for metric measure spaces, J. Funct. Anal. 275 (2018), no. 12, 3504-3569.

[37] S.-T. Yau, Harmonic functions on complete Riemannian manifolds, Comm. Pure Appl. Math. 28 (1975), 201-228.

[38] R. Ye, On the l-function and the reduced volume of Perelman. I, Trans. Amer. Math. Soc. 360 (2008), no. $1,507-531$.

[39] T. Yokota, Perelman's reduced volume and a gap theorem for the Ricci flow, Comm. Anal. Geom. 17 (2009), no. 2, 227-263.

[40] T. Yokota, On the asymptotic reduced volume of the Ricci flow, Ann. Global Anal. Geom. 37 (2010), no. 3, 263-274.

[41] Q. S. Zhang, Some gradient estimates for the heat equation on domains and for an equation by Perelman, Int. Math. Res. Not. 2006, Art. ID 92314, 39 pp.

Cooperative Faculty of Education, Utsunomiya University, 350 Mine-Machi, Utsunomiya, 321-8505, JAPAN

Email address: kunikawa@cc.utsunomiya-u.ac.jp

Department of Mathematics, Saitama University, 255 Shimo-Okubo, Sakura-ku, SaitamaCity, Saitama, 338-8570, Japan

Email address: ysakurai@rimath.saitama-u.ac.jp 